\title{
Nonlinear control of HIV-1 infection with a singular perturbation model
}

\author{
M. Barão ${ }^{\text {a }}$, J.M. Lemos ${ }^{\text {b,* }}$ \\ ${ }^{a}$ INESC-ID/University of Évora, Lisboa, Portugal \\ ${ }^{\mathrm{b}}$ INESC-ID/IST, Lisboa, Portugal
}

Received 28 February 2007; received in revised form 24 May 2007; accepted 25 July 2007

Available online 6 September 2007

\begin{abstract}
Using a singular perturbation approximation, a nonlinear state-space model of HIV-1 infection, having as state variables the number of healthy and infected CD4+T cells and the number of virion particles, is simplified and used to design a control law. The control law comprises an inner block that performs feedback linearizing of the virus dynamics and an outer block implementing an LQ regulator that drives the number of virion particles to a number below the specification. A sensitivity analysis of the resulting law is performed with respect to the model parameter to the infection rate, showing that the controlled system remains stable in the presence of significant changes of this parameter with respect to the nominal value. (C) 2007 Elsevier Ltd. All rights reserved.
\end{abstract}

Keywords: HIV-1; Feedback linearizing control; Stability robustness; LQ control; Singular perturbations

\section{Introduction}

Strategies for counteracting HIV infection designed using control methods are receiving an increased attention. Detailed studies that combine modelling analysis with clinical results show that the initial infection phase may be represented using simple nonlinear state models [1]. This fact boosted the production of an increasing number of papers where therapy strategies are derived from control principles. Examples include nonlinear control based on Lyapunov methods and on the use of decomposition in strict feedback form with backstepping [2], state drive using bang-bang control [3], adaptive control [4], optimal control [5], predictive control [6] as well as model based feedback [7]. In [8] various methods based on time-delay feedback control are shown, via Lyapunov function methods, to stabilize an HIV-1 model similar to the one considered in the present paper. In [9] a HIV-1 infection control strategy based on nonlinear geometric control (feedback linearization) is developed.

\footnotetext{
* Corresponding author.

E-mail addresses: mjsb@ramses.inesc.pt (M. Barão), jlml@inesc.pt (J.M. Lemos).
}

A straightforward approach to the design of a controller to regulate the state of a nonlinear system consists in obtaining an approximate linear model around the equilibrium point considered using Taylor series approximations and then to design a state feedback controller that drives the state increments with respect to the equilibrium to zero. Although simple, this method has the drawback of requiring that the initial conditions are close to the equilibrium for the approximation to be valid, being difficult to establish stability results. Furthermore, if the linearized system is not controllable, it may not be possible to design adequately the state feedback. This is the case of the model of HIV-1 infection considered hereafter around the equilibrium corresponding to an healthy person. If this approach is followed, the linearization must then be performed around the equilibrium point corresponding to infection and the state feedback controller should thus drive the state away from it [10], with the risk of becoming unstable due to the neglected higher order terms of the model.

Opposite to this approach, feedback linearization [11] aims at exactly cancelling the nonlinearities using a nonlinear static feedback. This results in a transformed model that is exactly linear in a region around the equilibrium point to which a linear regulator may then be applied. In this region, that is usually larger than the one resulting from Taylor approximation methods, stability of the closed loop is ensured. 
This paper proposes a strategy that combines model reduction using a simple singular perturbation approximation, feedback linearization and LQ regulation based on state feedback. As mentioned above, feedback linearization methods for designing HIV-1 therapy have also been considered in [9]. However, while in [9] the manipulated variable is assumed to enter linearly in the model, in this paper, the dependence on control is nonlinear, requiring a more complicated algebra, but representing in a more accurate way the effect of anti-retroviral drugs. Furthermore, in this paper, a stability robustness study is performed with respect to model parameter variation. Due to the wide variability of the dynamics associated to different patients the capacity of a controller to stabilize models that are different from the nominal one is quite important.

It should be remarked that the present paper, as well as the references quoted above, forms just one step towards the application of control techniques to the design of HIV-1 infection therapy. Indeed, in the actual clinical practice, the drugs currently used for treatment of HIV-1 infection are neither continuously infused nor is the virus concentration measured in permanence. The sampling of the controllers designed is therefore required, a subject that deserves attention on its own from the point of view of systems and control.

The paper is organized as follows. After this introduction, the reduced complexity model is derived in Section 2. Section 3 characterizes reduced model properties, viz. equilibrium points and controllability. As part of the discussion made on controllability, it is shown that the model draws to the conclusion that an infection can never be completely eliminated. Section 4 presents feedback linearization and Section 5 the control strategy. Section 6 draws conclusions. The mathematical details of the transformations involved in feedback linearization are presented in Appendices A and B as well as the proof of an equivalence result of the weights when LQ control is combined with feedback linearization.

\section{HIV-1 dynamic model}

The model used to describe the HIV-1 infection [10] is a deterministic one-compartment model with the following three state variables:

$x_{1}$ Concentration of healthy cells.

$x_{2}$ Concentration of infected cells.

$x_{3}$ Concentration of virions(free virus particles).

The equations connecting these variables read as follows:

$\dot{x}_{1}=s-d x_{1}-\left(1-u_{1}\right) \beta x_{1} x_{3}, \quad \dot{x}_{2}=\left(1-u_{1}\right) \beta x_{1} x_{3}-\mu x_{2}$,

$\dot{x}_{3}=\left(1-u_{2}\right) k x_{2}-c x_{3}$.

In the first equation, $s$ represents the production rate of healthy cells, the coefficient $d$ the natural death of the cells and $\beta$ the infection rate coefficient. The infection rate of healthy cells is proportional to the product of healthy cells $x_{1}$ and free virus $x_{3}$. This process can be influenced by drugs (Reverse Transcriptase Inhibitors-RTI) that reduce the virus performance. This influence is represented by the manipulated variable $u_{1}$, in which
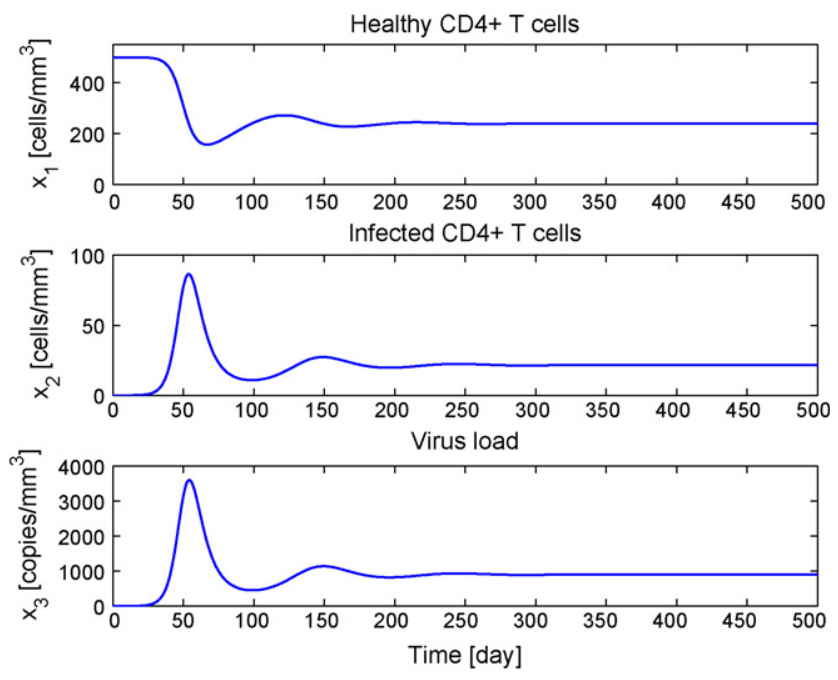

Fig. 1. Time response to an infection without medication.

$u_{1}=0$ corresponds to absence of drug and $u_{1}=1$ to a drug efficiency in preventing infection of $100 \%$. Actually, with the available drugs, the efficiency is below $100 \%$, and $u_{1}$ is constrained to the interval $\left[0, u_{\max }\right]$ with $u_{\max }<1$. The second equation comprises two terms that represent, respectively, the transition of healthy cells to infected cells and the death of infected cells, with $\mu$ the death coefficient.

An infected cell liberates free virus. This process is represented in the third equation, where the first term represents the liberation of virus by infected cells and the second the "death" of free virus with $c$ the corresponding coefficient. The manipulated variable $u_{2}$ represents the action of drugs (Protease Inhibitors-PI) that prevent infected cells to produce free virions.

Fig. 1 shows the transient time response to an HIV-1 infection. The parameters used [10] are the ones of Table 1. The initial conditions correspond to an healthy person infected with a virus concentration of 1 copy per $\mathrm{mm}^{3}$.

As can be seen, during an initial phase of the illness, lasting for about 30 days, the concentration of infected cells and free virus in the body is very small. After this period, a fast growth of the concentration of virus and infected cells is noticed, together with a major decrease of healthy CD4+T cells. After 6 months the infection stabilizes, and its kept in an approximated steady state for a period lasting between 2 and 10 years. After this period, through a mechanism not modelled in (1), the number of healthy CD4+T cells is drastically reduced and the patient develops AIDS.

Table 1

Model parameters

\begin{tabular}{lcl}
\hline Parameter & Value & Units \\
\hline$d$ & 0.02 & $\mathrm{~s}^{-1}$ \\
$k$ & 100 & $\mathrm{~s}^{-1}$ \\
$s$ & 10 & $\mathrm{~mm}^{-3} \mathrm{~s}^{-1}$ \\
$\beta$ & $2.4 \times 10^{-5}$ & $\mathrm{~mm}^{3} \mathrm{~s}^{-1}$ \\
$\mu$ & 0.24 & $\mathrm{~s}^{-1}$ \\
$c$ & 2.4 & $\mathrm{~s}^{-1}$ \\
\hline
\end{tabular}




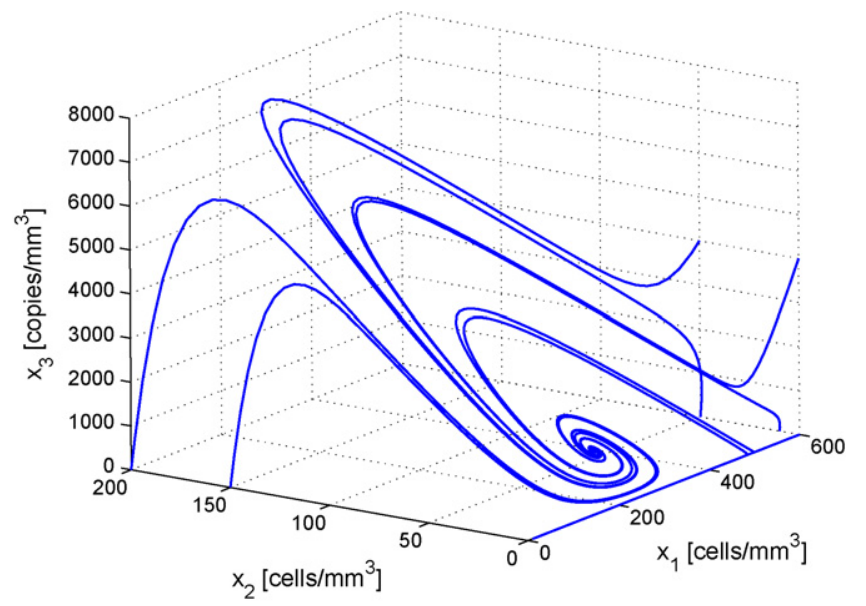

Fig. 2. State trajectories, starting from different initial conditions.

Fig. 2 shows some state trajectories obtained with different initial infection conditions. Although not all the initial conditions correspond to realistic infections, this plot provides insight into the type of dynamic behavior of (1). It is seen that the trajectories approach fast a plane before converging in spiral to an equilibrium point. This observation reveals the existence of two time scales for the system states, one fast and one slow, and suggests the use of singular perturbation methods. After the extinction of the fast transient, the variables $x_{2}$ and $x_{3}$ become approximately proportional. Fig. 3 shows the relation $x_{2} / x_{3}$, where it is apparent that, after a transient lasting about 2 or 3 days, the quotient of these variables is close to 0.024 with an initial small deviation.

\subsection{Reduced dynamic model}

By looking at the third state equation in $x_{3}$ it is seen that this defines a stable linear system with input $x_{2}$ and time constant $\tau_{\mathrm{v}}=1 / c \approx 0.42$ days. The equilibrium corresponds to the situation in that $\dot{x}_{3}=0$, implying

$x_{3}=\left(1-u_{2}\right) \frac{k}{c} x_{2}$

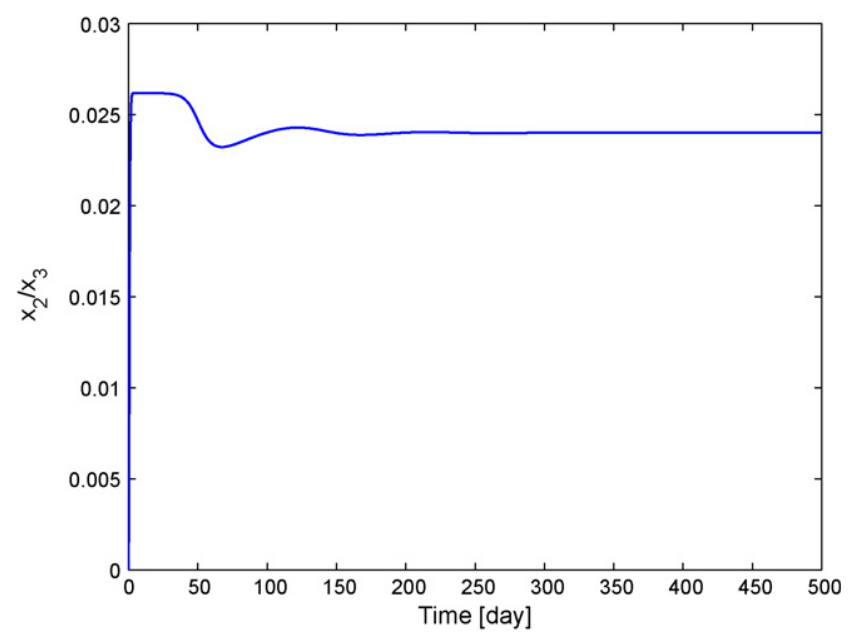

Fig. 3. Time evolution of the relation $x_{2} / x_{3}$.
Since the equation for $x_{3}$ is stable and converges fast to the equilibrium, the controller does not need to control this state explicitly and the model may be reduced to second order.

Replacing (2) in the state model (1), and assuming just one manipulated variable, yields the reduced second order model:

$$
\begin{aligned}
& \dot{x}_{1}=s-d x_{1}-(1-u) \frac{\beta k}{c} x_{1} x_{2}, \\
& \dot{x}_{2}=(1-u) \frac{\beta k}{c} x_{1} x_{2}-\mu x_{2} .
\end{aligned}
$$

\section{Model properties}

\subsection{Equilibrium points}

The analysis of equilibrium points and corresponding stability properties of the full model (1) has been performed in [10]. The reduced model is considered hereafter.

In the absence of therapy, $u=0$, the reduced model has as equilibrium points the solutions of the algebraic equations

$0=s-d x_{1}-(1-u) \frac{\beta k}{c} x_{1} x_{2}$

$0=(1-u) \frac{\beta k}{c} x_{1} x_{2}-\mu x_{2}$

with respect to the state variables $x_{1}$ and $x_{2}$. These equilibrium points are

$x_{1}=\frac{s}{d}, \quad x_{2}=0$

corresponding to an healthy person, and

$x_{1}=\frac{\mu c}{\beta k(1-u)}, \quad x_{2}=\frac{s}{\mu}-\frac{d c}{\beta k(1-u)}$

corresponding to an infected individual.

The stability analysis of these equilibrium points is made by computing the eigenvalues of the Jacobian matrix $\tilde{A}=\partial f / \partial x$, given by

$\tilde{A}=\left[\begin{array}{ll}-d-\frac{\beta k}{c} x_{2} & -\frac{\beta k}{c} x_{2} \\ \frac{\beta k}{c} x_{2} & \frac{\beta k}{c} x_{1}-\mu\end{array}\right]_{x=x_{\mathrm{eq}}}$

By using the model parameters of Table 1 , the results of Table 2 are obtained. These results are similar to the ones obtained for the full model, but in which the fast mode is absent.

\subsection{Controlability}

The reduced nonlinear model (3), may also be written as

$\dot{x}=f(x)+g(x) u$ 
Table 2

Stability of the equilibrium points of the reduced model

\begin{tabular}{ll}
\hline Equilibrium point & {$[240.000021 .6667]^{\mathrm{T}}$} \\
Eigenvalues & $-0.0208 \pm 0.0690 \mathrm{j}$ \\
Stability & Asymptotically stable \\
Equilibrium point & {$[500.00000 .0000]^{\mathrm{T}}$} \\
Eigenvalues & $-0.0200,0.2600$ \\
Stability & Unstable
\end{tabular}

with the vector functions $f$ and $g$ defined as

$f:=\left[\begin{array}{l}s-d x_{1}-\frac{\beta k}{c} x_{1} x_{2} \\ \frac{\beta k}{c} x_{1} x_{2}-\mu x_{2}\end{array}\right]$

$g:=\frac{\beta k}{c} x_{1} x_{2}\left[\begin{array}{l}1 \\ -1\end{array}\right]$

Model (9) may be linearized around an equilibrium point $\left(x_{1}^{*}, x_{2}^{*}\right)$ yielding a linear model

$\dot{\tilde{x}}=A \tilde{x}+B u$

where $\tilde{x}$ is the increment of the state with respect to the equilibrium. The corresponding controllability matrix is given by

$\mathcal{C}=\left[\begin{array}{ll}B & A B\end{array}\right]=\frac{\beta k}{c} x_{1} x_{2}\left[\begin{array}{ll}1 & -d-\frac{\beta k}{c}\left(x_{2}-x_{1}\right) \\ -1 & \frac{\beta k}{c}\left(x_{2}-x_{1}\right)+\mu\end{array}\right]$

and it is seen that

$\operatorname{rank}(\mathcal{C})=\left\{\begin{array}{lll}2 & \text { if } d \neq \mu, \quad x_{1} \neq 0, & x_{2} \neq 0 \\ 1 & \text { if } d=\mu, \quad x_{1} \neq 0, & x_{2} \neq 0 \\ 0 & \text { if } x_{1}=0 \text { or } x_{2}=0 & \end{array}\right.$

Since $d \neq \mu$ and $x_{1}>0$, it is concluded that the controllability matrix has rank 2 for $x_{2} \neq 0$ (infected individual) and rank 0 for $x_{2}=0$ (healthy individual).

When the linearized system is not controllable, it may still be possible to control the nonlinear system. The controllability analysis of a nonlinear system is much more complex than in the linear case and, since there are no global results, the controllability in the nonlinear case can only be studied locally.

The reachable set $R^{V}\left(x_{0}, T\right)$, with $T$ finite, is defined as the set of states $x$ for which there is an admissible control input $u(t)$ that drives the state $x(t)$ from the initial state $x(0)=x_{0}$ to the final state $x(T)=x$, satisfying simultaneously $x(t) \in V, t \in[0, T]$. It is proved in [11] that if, for a given $x_{0}$, the Lie Algebra $\mathcal{C}\left(x_{0}\right)$ generates a space of dimension $n$ then, in any neighborhood $V$ of $x_{0}$ and $T>0$, the reachable set $R_{T}^{V}\left(x_{0}\right)=\cup_{\tau<T} R^{V}\left(x_{0}, \tau\right)$ contains a non-empty open subset of the state pace. In this case the system is said to be locally reachable from $x_{0}$.

In (14) it can be verified that for $x_{2} \neq 0$ the local linearization is controllable, and the doubt remains only on the situation in which $x_{2}=0$. The following question may then be posed: Is there any state $x$ with $x_{2} \neq 0$ such that the reachable set from that state contains a final state with $x_{2}=0$ ? In clinical terms, this question reads: Is it possible that an individual, once infected, gets again rid of the infection? The answer to this question is negative, as shown below.

Computing the Lie Algebra in all the points of the state space with $x_{2} \neq 0$ it is seen that the accessibility rank condition is 2 (it is enough to verify that $f(x)$ and $g(x)$ are linearly independent), and hence this region is locally reachable. On the other way, computing the Lie Algebra for the states $x=\left(x_{1}, 0\right)$, and since, for these states

$f(x)=\left[\begin{array}{ll}10-0.02 x_{1} & 0\end{array}\right]^{\prime}$

$g(x)=\mathbf{0}$

all the Lie brackets of higher order vanish and the rank reachability condition is 1 . The nonlinear system is then locally nonreachable in the region $\left\{x \mid x_{2}=0\right\}$, meaning that it is not possible to pass an healthy individual to the state of being infected by drug administration (a fact that is expected).

Much more interesting is to prove that there is not a trajectory in the opposite sense. By considering the second state equation in (3)

$\dot{x}_{2}=\underbrace{(1-u)}_{>0} \underbrace{\frac{\beta k}{c}}_{>0} \underbrace{x_{1} x_{2}}_{\geq 0}-\mu x_{2}$

it is observed that, since the first term in the right-hand-side is non-negative, then $x_{2}$ never vanishes and, hence, it is never possible to eliminate the infection.

\section{Feedback linearization}

System (3) is not linearizable by performing a state transformation only. However, by the combined use of the transformations

$u=\alpha(x)+\beta(x) v$

$z=S(x)$

the following linear model is obtained

$\dot{z}=A z+B v$

with

$A=\left[\begin{array}{ll}0 & 1 \\ 0 & 0\end{array}\right], \quad B=\left[\begin{array}{l}0 \\ 1\end{array}\right]$

Fig. 4 shows a block diagram of these transformations. The manipulated variable $v$ in the transformed model is called "virtual" because it has only mathematical existence, in opposition to $u$, that has the physical meaning of being the drug dosage actually applied to the patient. Eq. (18) allows to compute the actual drug dose $u$ such that between $v$ and $z$ there is a linear relationship to which linear control techniques may then be applied. 


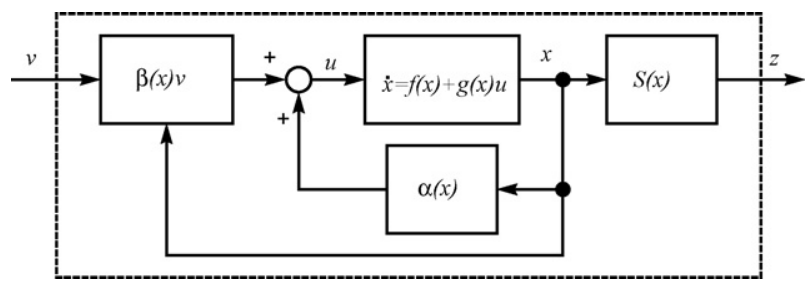

Fig. 4. Feedback linearization.

In the case at hand, it is shown in Appendix A that the transformations performing linearization are

$\beta(x)=\frac{c}{\beta k x_{1} x_{2}(\mu-d)}$

$\alpha(x)=\frac{-d s+d^{2} x_{1}+\mu^{2} x_{2}+(d-\mu)(\beta k / c) x_{1} x_{2}}{(d-\mu)(\beta k / c) x_{1} x_{2}}$

$S(x)=\left[\begin{array}{l}\varphi(x) \\ s-d x_{1}-\mu x_{2}\end{array}\right]$

with $\varphi(x)$ given by (47) in Appendix A. With these transformations, the system in a region of state space around the equilibrium (7) is transformed exactly in the linear system (21). Fig. 5 shows the response of the linearized system to a rectangular virtual input (i.e. the input $v$ before the transform). This simulation assumes that all parameters are exactly known and the transform is applied to the full model (1). Remark that the full model (1) is selected to perform the simulation in order to test the validity of the conclusions obtained with the reduced model.

It is remarked that, during the initial period, the signal $v(t)$ is negative and causes $u(t)$ to saturate. For that reason, the linearized system does not behave like a double integrator during that interval of time. Indeed, with a good approximation, $z_{2}$ is a
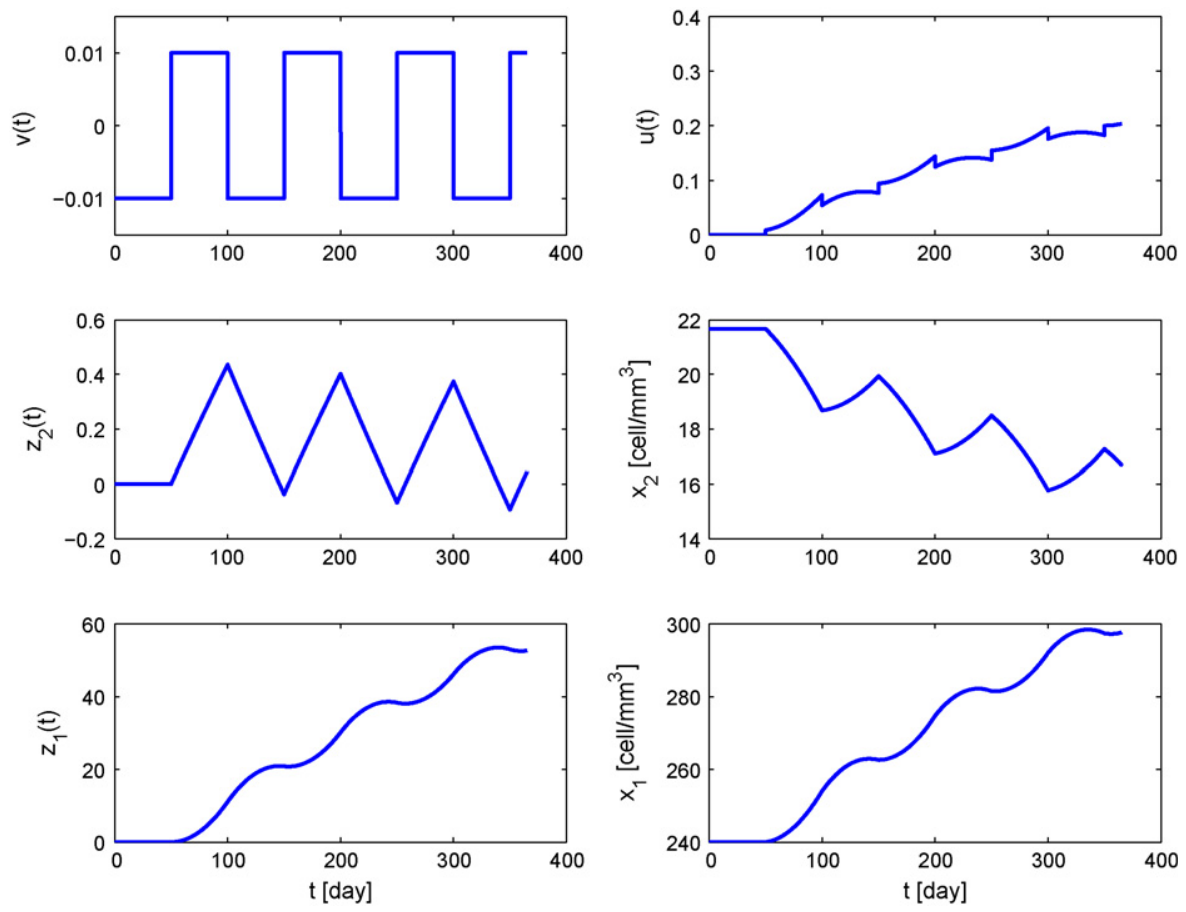

triangular signal corresponding to the integral of the square signal $v$ (that is squared) and $z_{2}$ is made of parabolic segments, corresponding to the integral of the triangular shaped $z_{2}$.

\section{HIV-1 viral load control}

This section shows how to develop a control law for the system linearized by feedback. This is done both under the hypothesis of perfect (in the first stage) and partial knowledge of the system parameters.

\subsection{Control with known parameters}

Assume that the concentration of infected cells $x_{2}$ is to be driven to a reference value $r$ and kept there. At the equilibrium defined by $x_{2}=r$ one has by equating the derivatives to zero in (3)

$(1-u)=\frac{\mu d c}{\beta k(s-\mu r)}$

and

$x_{1}=\frac{s-\mu r}{d}$

In terms of the linearized system (that operates with transformed variables) this results in the equilibrium point $z=S(x)$, i.e.:

$\left[\begin{array}{l}z_{1} \\ z_{2}\end{array}\right]=\left[\frac{s-\mu r}{d}+r+\frac{c}{\beta k}(d-\mu)-\frac{s}{\mu}\right]=: T(r)$

It is then possible to design a LQ controller, using the linearized dynamics, that keeps the system at the desired reference value $r$.
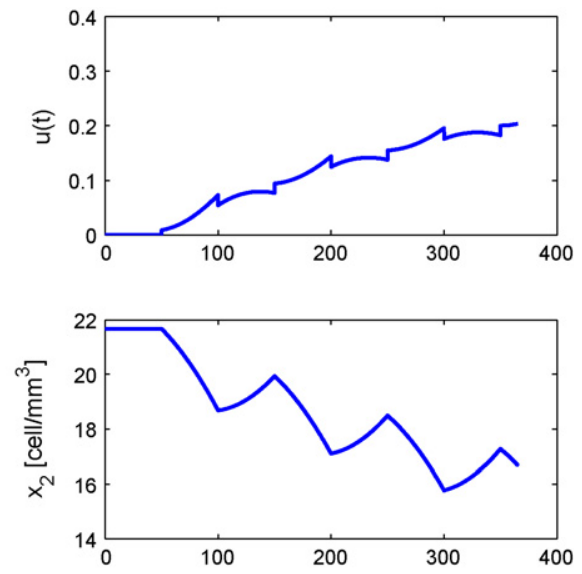

Fig. 5. Time response of the linearized system to a virtual rectangular system (virtual signal on the left, actual signal on the right). 

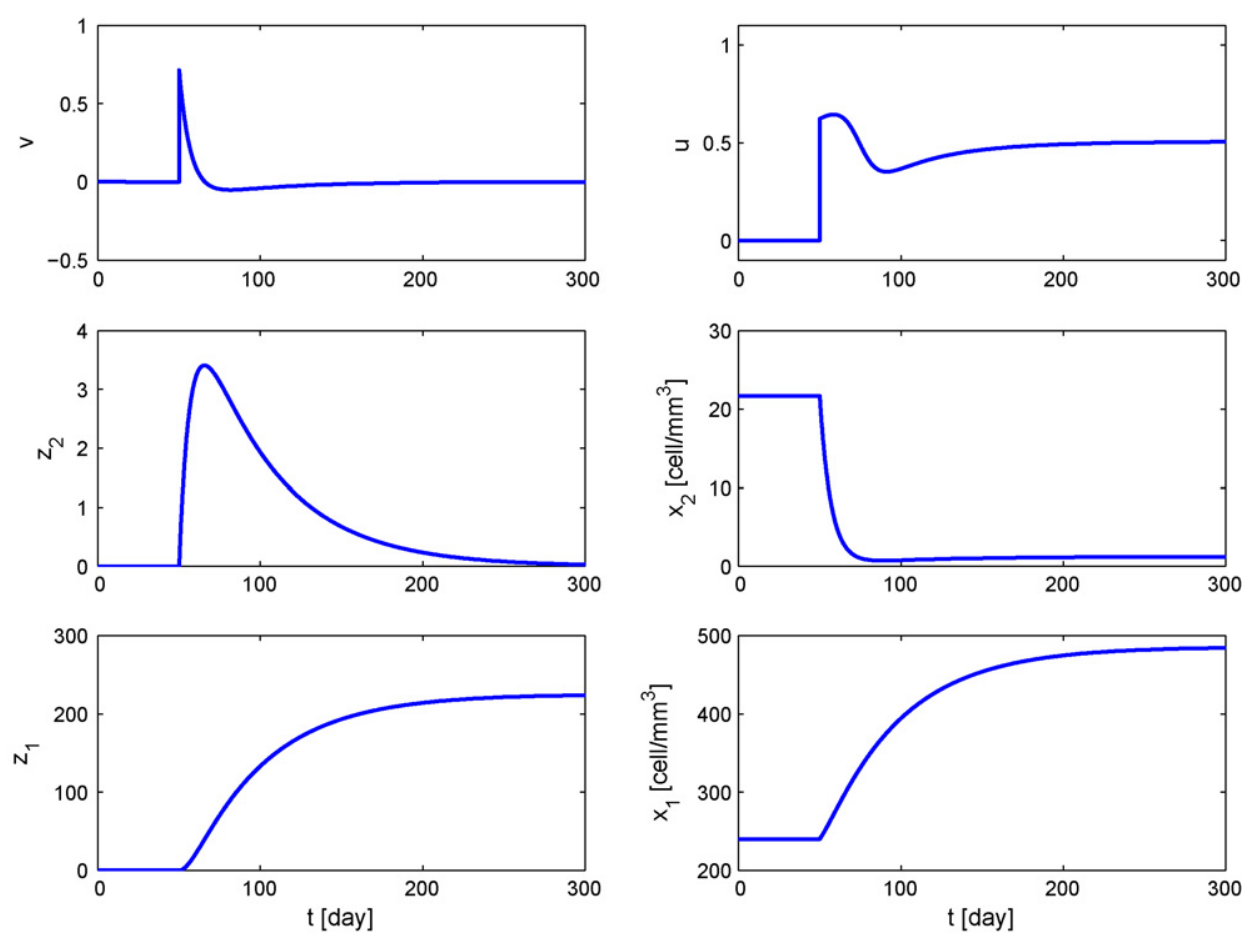

Fig. 6. Changing the reference in the number of infected cells.

The transformation $T(r)$ allows to compute the equilibrium point in terms of the variables $\left(z_{1}, z_{2}\right)$. The controller is designed by minimizing the quadratic cost:

$J=\int_{0}^{+\infty} z^{\mathrm{T}} Q_{z} z+\rho v^{2} \mathrm{~d} t$

where $Q_{z}$ and $\rho$ adjust the contribution of the variables $z(t)$ and $v(t)$. Since these variables are virtual (corresponding to transformed states) it is difficult to develop heuristic choices of their values. Thus it was decided to adjust the weights $Q_{x}$ for the state variables $x$ and then compute the corresponding $Q_{z}$. Using a
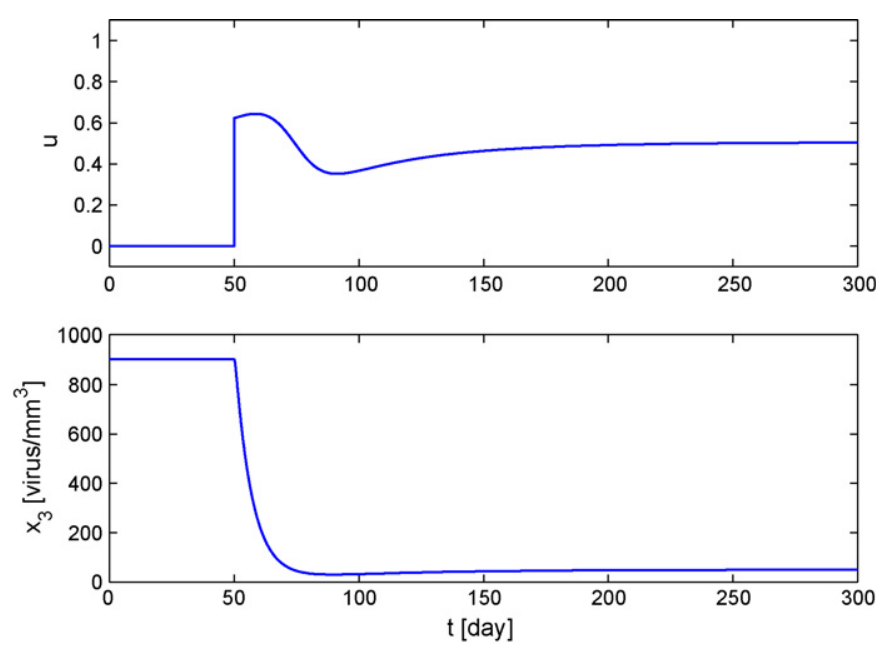

Fig. 7. Evolution of viral load. linear approximation, it is shown in Appendix B that

$Q_{z}=\left(\frac{\partial S^{-1}}{\partial z}\right)^{\mathrm{T}} Q_{x}\left(\frac{\partial S^{-1}}{\partial z}\right)$

With the following choice of the weights

$Q_{x}=\left[\begin{array}{cc}0.01 & 0 \\ 0 & 23\end{array}\right], \quad \rho=10^{3}$

the results shown in Figs. 6 and 7 are obtained. These weights are "tuning knobs" that allow the designer to adjust the relative
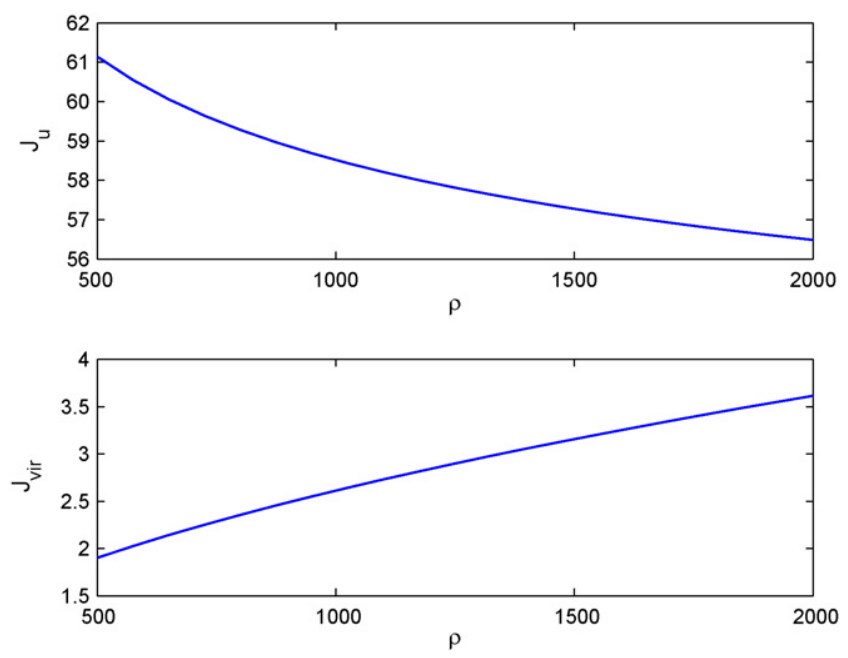

Fig. 8. Selection of the weight $\rho$. 


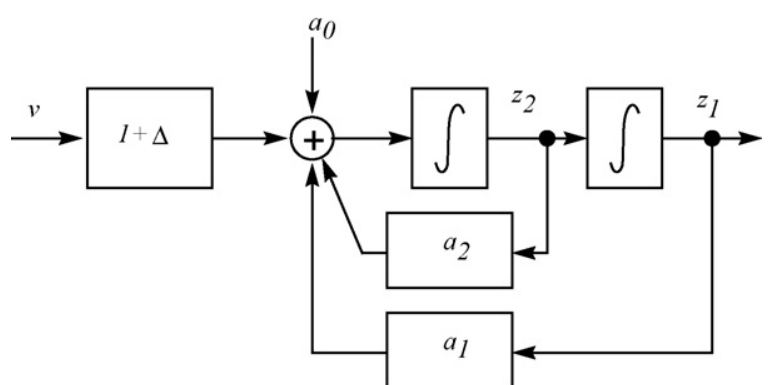

Fig. 9. Quasi-linearized system (remark that with $\Delta=0$ the double integrator is recovered).

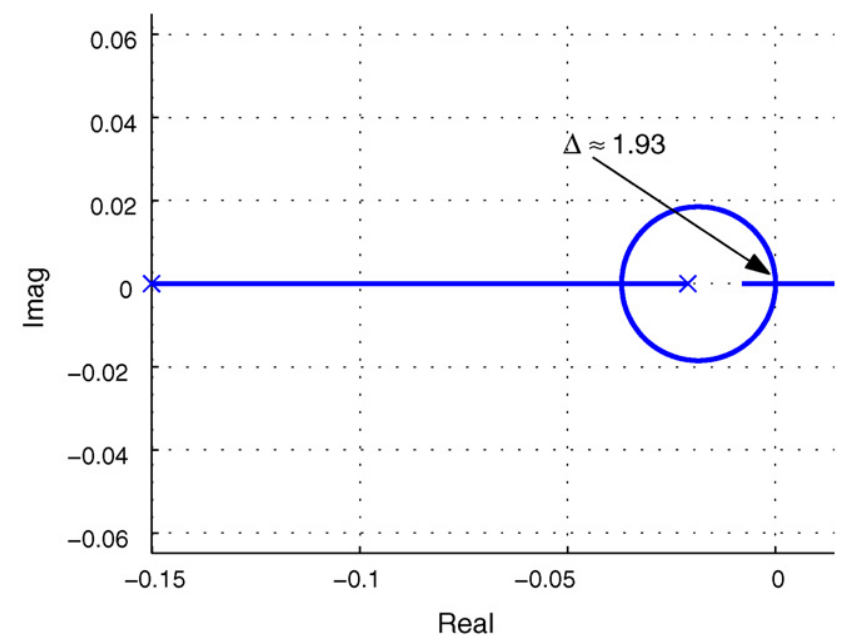

Fig. 10. Root-locus of the poles of the closed loop system (with the LQ regulator), as a function of the uncertainty $\Delta \in[0,2]$.
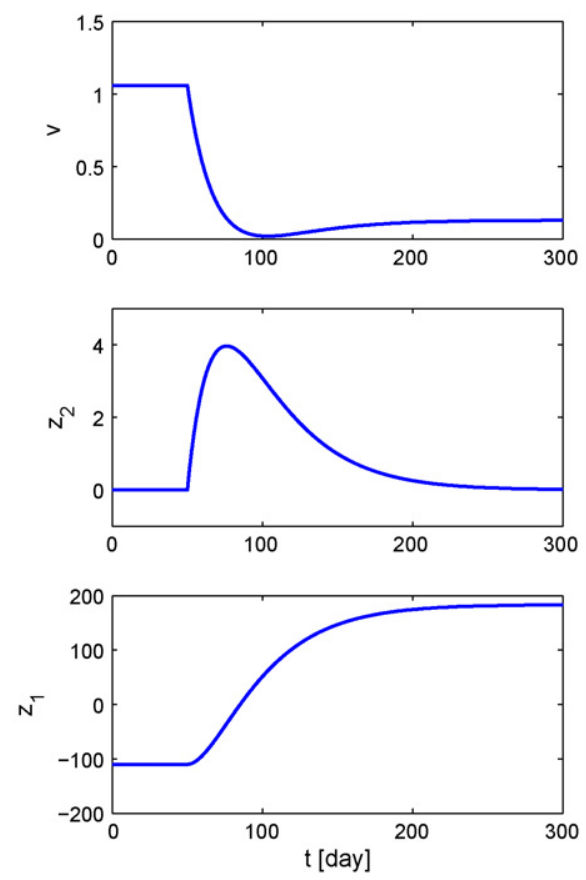

importance of the state variables and the drug usage. The simulation has been performed using the full, third order, model.

Fig. 6 shows in the three left graphics the variables of the linearized system (virtual input $v$ and states $z_{1}, z_{2}$ ), and on the three graphics of the right the actual variables (input $u$ and states $x_{1}, x_{2}$ ) with the above choice of weights. Fig. 7 shows the concentration of free virus. Its value decays fast, such as the one of infected cells, as shown in Fig. 6. The specification consists in reducing the number of virus to 50 copies per $\mathrm{mm}^{3}$ in a period of less then 50 days.

In order to provide an overall idea of the influence of $\rho$ on control performance, Fig. 8 plots

$J_{\mathrm{u}}:=\int_{50}^{300} u^{2}(t) \mathrm{d} t$

and

$J_{\mathrm{vir}}:=\gamma \int_{50}^{300}\left(x_{3}(t)-r\right)^{2} \mathrm{~d} t, \quad \gamma=10^{-6}, \quad r=50$

as a function of $\rho$. Decreasing $\rho$ leads to a smaller viral load integrated over time, but to bigger drug dose administration. The choice $\rho=10^{-3}$ was selected as a possible compromise.

\subsection{Control with uncertain parameters}

Consider now the problem of control design by feedback linearization in the presence of structured uncertainty in the model. Assume that there is multiplicative uncertainty in parameter $\beta$, i.e., the actual system uses $\beta(1+\Delta)$, with $\Delta$ unknown, while the linearization assumes the nominal model $(\Delta=0)$. Thus, the actual model is described by the state
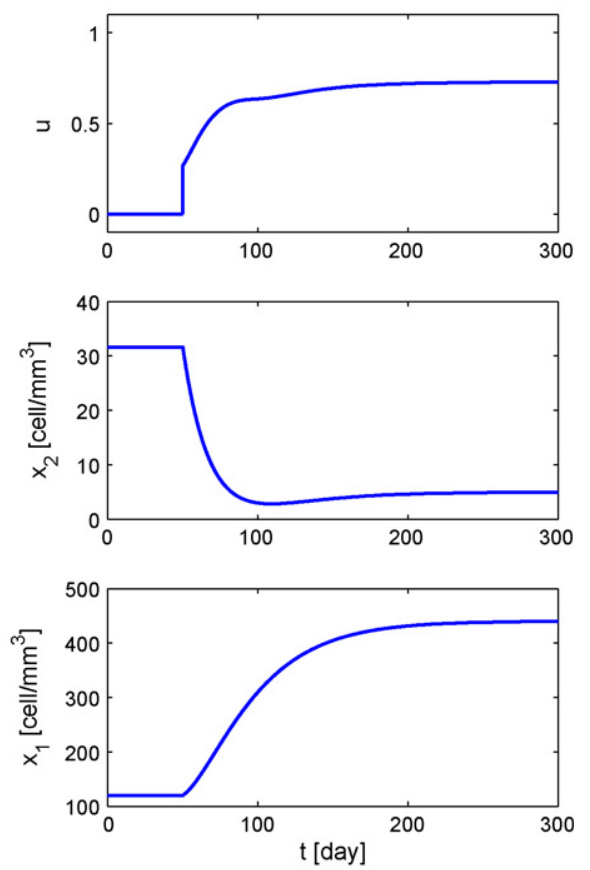

Fig. 11. Control performance with uncertainty on the parameter $\beta(\Delta=1$, i.e., the true $\beta$ is twice the nominal value). 

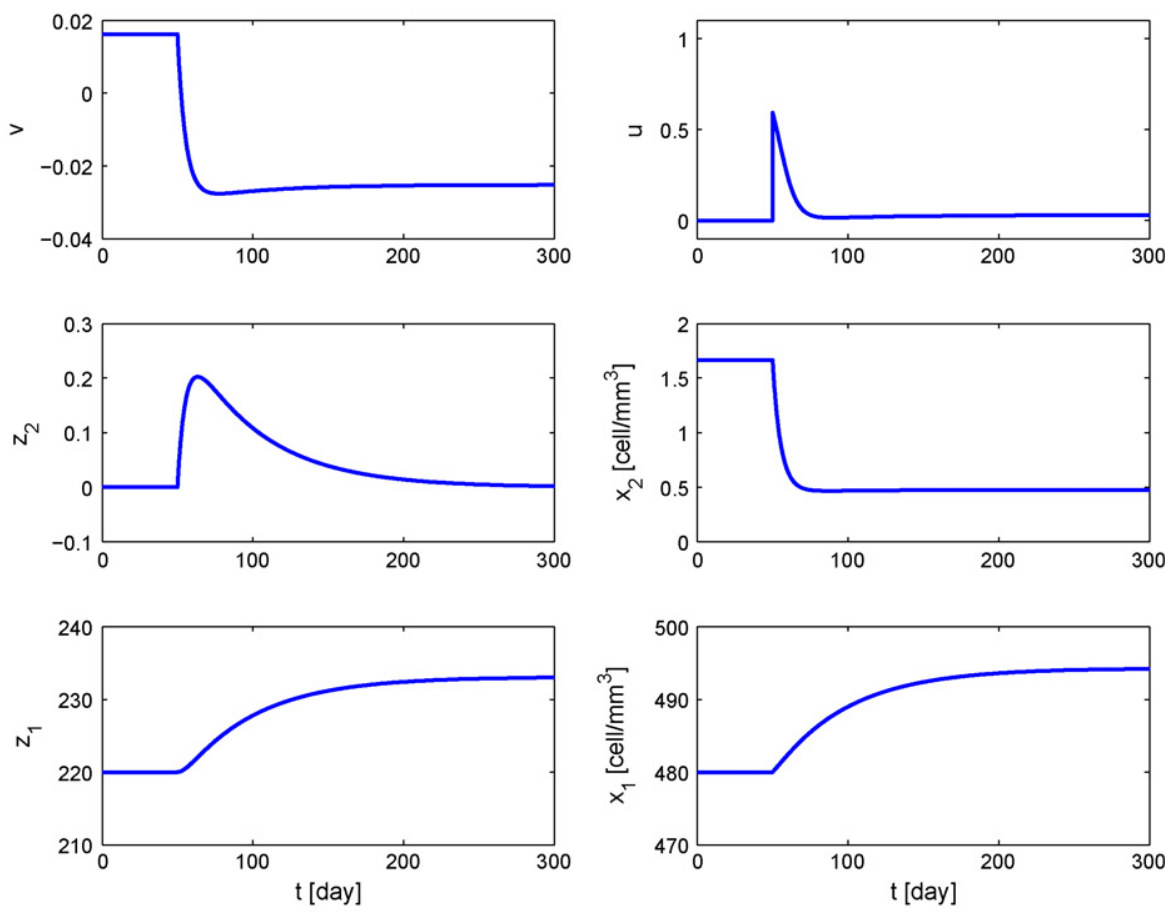

Fig. 12. Control performance with uncertainty on the parameter $\beta(\Delta=-0.5$, i.e., the true $\beta$ is half of the nominal value).

equation

$\dot{x}=f(x, \Delta)+g(x, \Delta) u$

while the linearization uses the nominal model given by

$\dot{x}=f(x, 0)+g(x, 0) u$

By applying the feedback linearization transformations yielded by the nominal model in the actual model, it is expected that the final result is no longer exactly a double integrator. In this case the following "quasi-linearized system" is obtained:

$\dot{z}_{1}=z_{2}, \quad \dot{z}_{2}=v+\Delta\left(a_{0}+a_{1} z_{1}+a_{2} z_{2}+v\right)$

where

$a_{0}=\frac{d \mu c}{\beta k}-s, \quad a_{1}=d \mu, \quad a_{2}=d+\mu$

Fig. 9 shows the structure of the system thereby obtained.

The additional term due to the uncertainty $\Delta$ does not change the linear characteristic of the quasi-linearized system. There is only a pole displacement, variation of the loop gain and an additive disturbance at the input.

In closed-loop, and with the LQ regulator designed above the closed loop poles are as in Fig. 10.

By applying standard root-locus methods, it is possible to compute analytically that the closed-loop remains stable for values of $\Delta \in]-1,1.93[$. This ensures the robustness of the controller design with respect to uncertainty on parameter $\beta$. For the sake of illustration, Figs. 11 and 12 show simulations with $\Delta=1$ and $\Delta=-0.5$. These values for $\Delta$ have been selected in the mid positive and negative range of $\Delta$ that ensure stability.
Although the system remains stable under an error in the nominal value for $\Delta$, the final values reached are not coincident with the reference. This is caused by two types of factors:

1. The quasi-linearized system is no longer a double integrator. In particular, as seen in the block diagram of Fig. 9, there is a change in gain due to the cascaded block $1+\Delta$, an additive disturbance associated with $\Delta a_{0}$ is present and the poles are shifted from the origin by the disturbance feedback terms $\Delta a_{1}$ and $\Delta a_{1}$.

2. Furthermore, the transform from the space $\left(x_{1}, x_{2}\right)$ to the virtual space $\left(z_{1}, z_{2}\right)$ depends on the nominal value of $\beta$. Hence, the virtual reference "seen" by the controller becomes wrong.

\section{Conclusion}

The paper studies nonlinear control of HIV-1 infection. Using a simple singular perturbation approximation, a reduced model is first obtained and used to show that it is not possible to completely eliminate the infection resorting only to the available manipulated variable.

In order to constrain the infection level to be below a specified level (50 virus copies/ $\mathrm{mm}^{3}$ in the plasma) a nonlinear controller is proposed, comprising two nested loops. The inner loop performs the exact feedback linearization of the system, while the outer loop is a LQ regulator.

A sensitivity study of the effect of the variations of one of the parameters has been performed, showing that the closed-loop system remains stable within reasonably large bounds of uncertainty. 


\section{Acknowledgement}

Part of this work was performed under the framework of project DYNAMO —Dynamic modelling, control and optimization of metabolic networks, PTDC/EEA-ACR/69530/2006.

\section{Appendix A. Feedback linearization}

Consider the nonlinear system (9) that is assumed to be not equivalent to a linear system, in the sense that there is no diffeomorphism of the state that transforms it in a linear model. Applying the transformation $u=\alpha(x)+\beta(x) v$ to the input, yields the state equation

$$
\begin{aligned}
\dot{x} & =f(x)+g(x)(\alpha(x)+\beta(x) v) \\
& =\underbrace{(f(x)+g(x) \alpha(x))}_{\tilde{f}(x)}+\underbrace{g(x) \beta(x)}_{\tilde{g}(x)} v
\end{aligned}
$$

that may be written as the modified model

$\dot{x}=\tilde{f}(x)+\tilde{g}(x) v$.

If the modified model (36) is equivalent to a linear system, it is then possible to obtain a linear system by the combined application of the transforms (18), (19) yielding the linear model (20) that relates $v$ and $z$.

Not all nonlinear systems are linearizable using this procedure (known as feedback linearization). In [11] it is shown that the nonlinear system (9) with $f\left(x_{0}\right)=0$ and scalar input $u$ is feedback linearizable around the equilibrium $x_{0}$ if and only if the distributions $D_{i}$ defined by

$D_{i}=\operatorname{span}\left\{g(x), \operatorname{ad}_{f} g(x), \ldots, \operatorname{ad}_{f}^{i-1} g(x)\right\}$

verify the two following conditions:

$\operatorname{dim} D_{n}\left(x_{0}\right)=n$,

$D_{n-1}$ is involutive around $x_{0}$

In relation to the model (9) with $f$ and $g$ given by (10) and (11), the first condition results in

$$
\begin{aligned}
\operatorname{dim} D_{2}(x) & =\operatorname{rank}\left[g(x) \quad \operatorname{ad}_{f} g(x)\right] \\
& =\frac{\beta k}{c} x_{2} \operatorname{rank}\left[\begin{array}{cc}
x_{1} & s-\mu x_{1} \\
-x_{1} & -s+d x_{1}
\end{array}\right] \\
& =2 \text { for } x_{1}, x_{2} \neq 0 \text { and } \mu \neq d .
\end{aligned}
$$

and hence $\operatorname{dim} D_{2}\left(x_{0}\right)=2$. The second condition is also verified because $D_{1}=\operatorname{span}\{g(x)\}$ is involutive since $[g, g]=0 \in D_{1}$. The model is therefore feedback linearizable.

Since the conditions on $D-i$ are satisfied, there exists [11] a function $\varphi(x)$ that verifies the following three conditions:

$\varphi\left(x_{0}\right)=0$

$\left\langle\mathrm{d} \varphi, \operatorname{ad}_{f}^{k} g\right\rangle(x)=0, \quad k=0,1, \ldots, n-2$

$\left\langle\mathrm{d} \varphi, \operatorname{ad}_{f}^{n-1} g\right\rangle\left(x_{0}\right) \neq 0$
In terms of $\varphi(x)$, the linearizing transforms yielding (21) around the equilibrium state $x_{0}$ are given by [11]:

$\alpha(x)=-\left(L_{g} L_{f}^{n-1} \varphi(x)\right)^{-1} L_{f}^{n} \varphi(x)$

$\beta(x)=\left(L_{g} L_{f}^{n-1} \varphi(x)\right)^{-1}$

$z_{i}=L_{f}^{i-1} \varphi(x), \quad i=1,2, \ldots, n$

The function

$\varphi(x)=x_{1}+x_{2}-\frac{\mu c}{\beta k}-\frac{s}{\mu}+\frac{d c}{\beta k}$

satisfies the three conditions, in particular

1. Computing $\varphi(x)$ at the equilibrium $x_{0}$ given by point (7) yields $\varphi\left(x_{0}\right)=0$;

2. $\langle\mathrm{d} \varphi, g\rangle=(\partial \varphi(x) / \partial x) g(x)=0$;

3. $\langle\mathrm{d} \varphi,[f, g]\rangle=(\partial \varphi(x) / \partial x)[f, g]=(\beta k / c)(d-\mu) x_{1} x_{2} \neq 0$, para $x=x_{0}$.

Using $\varphi(x)$ as given by (47) and (44)-(46) yields the transformations (22)-(24).

The expression (47) for $\varphi(x)$ is obtained by noting that Condition 2 may be written as

$\left[\begin{array}{ll}\frac{\partial \varphi}{\partial x_{1}} & \frac{\partial \varphi}{\partial x_{2}}\end{array}\right]\left[\begin{array}{l}1 \\ -1\end{array}\right] \frac{\beta k}{c} x_{1} x_{2}=0$

and hence implies that $\varphi(x)$ satisfies the partial differential equation

$\frac{\partial \varphi}{\partial x_{1}}=\frac{\partial \varphi}{\partial x_{2}}$

whose solution is given by any differentiable function $\Phi$ of argument $x_{1}+x_{2}$ :

$\varphi\left(x_{1}, x_{2}\right)=\Phi\left(x_{1}+x_{2}\right)$

The simplest choice that also satisfies Condition 1 is given by (47).

\section{Appendix B. Weight selection}

Since $x=S^{-1}(z)$, the corresponding in $z$ to the quadratic form in $x$ is given by

$$
\begin{aligned}
& \left(x-x_{0}\right)^{\mathrm{T}} Q_{x}\left(x-x_{0}\right) \\
& \quad=\left(S^{-1}(z)-S^{-1}\left(z_{0}\right)\right)^{\mathrm{T}} Q_{x}\left(S^{-1}(z)-S^{-1}\left(z_{0}\right)\right)
\end{aligned}
$$

that is not, in general, a quadratic form in $z$.

Using the linear approximation

$$
S^{-1}(z) \approx S^{-1}\left(z_{0}\right)+\left.\frac{\partial S^{-1}}{\partial x}\right|_{z_{0}}\left(z-z_{0}\right)=S^{-1}\left(z_{0}\right)+\left[\begin{array}{ll}
1 & 1 \\
-d & -\mu
\end{array}\right] z
$$


and replacing (52) in (51), it follows that

$\left(x-x_{0}\right)^{\mathrm{T}} Q_{x}\left(x-x_{0}\right)=z^{\mathrm{T}}\left(\frac{\partial S^{-1}}{\partial z}\right)^{\mathrm{T}} Q_{x}\left(\frac{\partial S^{-1}}{\partial z}\right) z$

and hence (29) follows.

\section{References}

[1] A. Perelson, P. Nelson, Mathematical analysis of HIV-1 dynamics in vivo, SIAM Rev. 41 (1) (1999) 3-44.

[2] S. Gee, Z. Tian, T. Lee, Nonlinear control of a dynamic model of HIV-1, IEEE Trans. Biomed. Eng. 52 (3) (2005) 353-361.

[3] H. Chang, H. Shim, J.H. Seo, Control of immune response of HIV infection model by gradual reduction of drug dose, in: Proceedings of the IEEE Conference on Decision and Control, 2004.

[4] C.-F. Cheng, C.-T. Chang, Viral load analysis of a biodynamical model of HIV-1 with unknown equilibrium points, in: Proceedings of the 2004 IEEE Conference on Control Applications, Taipei, Paiwan, (2004), pp. 557-561.
[5] J.F. de Souza, M. Caetano, T. Yoneyama, Optimal control applied to the antiviral treatment of AIDS, in: Proceedings of the 39th IEEE Conference on Decision and Control, Sydney, Australia, (2000), pp. 48394844.

[6] R. Zurakowski, A. Teel, A model predictive control based scheduling method for HIV therapy, J. Theor. Biol. 238 (2006) 368-382.

[7] J. Alvarez-Ramirez, M. Meraz, J. Velasco-Hernandez, Feedback control of the chemotherapy of HIV, Int. J. Bifuraction Chaos 10 (9) (2000) 22072219.

[8] M.E. Brandt, G. Chen, Feedback control of a biodynamical model of HIV1, IEEE Trans. Biomed. Eng. 48 (7) (2001) 754-759.

[9] F.L. Biafore, C.E. D'Atellis, Exact linearization and control of a HIV-1 predator-prey model, in: Proceedings of the 2005 IEEE Engineering in Medicine and Biology 27th Annual Conference, Shanghai, China, September 1-4, (2005), pp. 2367-2370.

[10] I. Craig, X. Xia, J. Venter, Introducing HIV/AIDS education into electrical engineering curriculum at the University of Pretoria, IEEE Trans. Educ. 47 (1) (2004) 65-73.

[11] H. Nijmeijer, A. van der Schaft, Nonlinear Dynamical Control Systems, Springer-Verlag, 1990. 